\title{
AALUMO: A User Model Ontology for Ambient Assisted Living Services supported in Next-Generation Networks
}

\author{
P.A. Moreno M.E. Hernando $\quad$ E.J. Gómez
}

\begin{abstract}
Ambient Assisted Living (AAL) services are emerging as context-awareness solutions to support elderly people's autonomy. The context-aw are paradigm makes applications more user-adaptive. In this way, context and user models expressed in ontologies are employed by applications to describe user and environment characteristics. The rapid advance of technology allows creating context server to relieve applications of context reasoning techniques. Specifically, the Next Generation Networks (NGN) provides by means of the presence service a framework to manage the current user's state as well as the user's profile information extracted from Internet and mobile context. This paper propose a user modeling ontology for AAL services which can be deployed in a NGN environment with the aim at adapting their functionalities to the elderly's context information and state.
\end{abstract}

Keywords - context-aware applications, ambient assisted living, next-generation network, user model, ontology

\section{INTRODUCTION}

Nowadays, the world population is experiencing a progressive ageing due to medical and technological advances which improves the life expectancy. Therefore, an enormous interest is growing to use the information and communication technologies (ICT) to support elderly people to live independently for longer period in their home. Presently, Ambient Assisted Living (AAL) [1] is one of the significant ICT initiatives for elderly people with special needs. The AAL services belong to the context-aware paradigm in which computing technology becomes closely invisible embedded into everyday objects making services more personalized, adaptable, interactive and therefore useful.

Since context can be defined as "any information that can be used to characterize situation and specifies the elements that must be observed to model situation" [2], contextaware applications employ sensors and context sources to drive their performance in specific user's conditions or preferences. Thus, context modeling explains contexts and the relationships among the distributed heterogeneous contexts. Context and user models describe through formal and conceptual language user's aspects (profile, preferences, interest, education, profession or living conditions) as well as environment information to improve services' features.
Ontologies are used to represent context data of models, so systems and devices can interpret the semantics of processing information allowing interoperability in an AAL environment. Ontology-based languages and tools enable a formal analysis of the domain knowledge and promote contextual knowledge sharing and reuse in context-aware systems [3]. Ontology Web Language (OWL) allows to construct complex, graph-like hierarchies of user model concepts, which is especially important for ontology integration. Several ontologies as UserML, OntobUM or GUMO allow modeling user's context. The User Modeling Mark-up Language (UserML) models user in ubiquitous computing; and OntobUM integrates three ontologies: one for user, another for defining relations between applications and other to define the user-application interaction semantics. The General User Modeling Ontology (GUMO) is used for a uniform interpretation of distributed user models in intelligent Semantic Web enhanced environments [4].

With the rapid advance in technology, users can take advantage of the devices and services enriched with context information. The evolution of ICT toward an horizontal service platform, supporting anytime and anywhere access to information and knowledge-driven and context-aware decision making, can act as a driver toward the delivery of AAL services. A good amount of research has been conducted lately to develop integrated context management frameworks aimed at acting as context servers, relieving the final context-aware application of all context-management related operation [3]

With the appearance of Next-Generation Networks (NGN) telecom operators can leverage All-IP networks to design external service interfaces that integrate a diverse set of sources and context inference processes that are easily scalable, extendable, and robust at the same time [5]. NGN is based on the IP Multimedia Subsystem (IMS) architecture which supports context inference that enables multimedia application services to acquire rich user information from both Internet and mobile spheres regardless of the access network. The horizontality of IMS architecture provides a group of functions called "service enablers" that are common to many third-party applications and therefore should be built once and reuse many times. Therefore, context management can take advantage of user provisioning, man- 
agement and security features offered by the NGN infrastructure. Despite users are reluctant to give personal information to third parties, telco operators have traditionally been considered reliable and trustable. Thus, a telco is particularly suitable to offer context-aware services because it is an intermediary between the user, who gets access to the communication network, and software developers, who use the NGN to provide their enriched applications

Some related context-aware works employ ontological approaches to manage information in smart homes and health services [6] or to support ambient intelligence mobile services [7]. Some context-aware services use NGN infrastructure to build a map of user meaningful context status [8]; or a NGN service enabler to handle context information of device, network, user, and position to decide the appropriate services for a user [9]. Finally,[10] propose the creation of a context knowledge layer in the IMS layered architecture with the aim at helping $3^{\text {rd }}$ party service providers to create personalized services considering user's context (preferences, device, networks or service).

The AAL user model ontology proposed in this work is aimed at being employed by an IMS architecture in order to provide third party AAL applications with personalization and adapting functionalities considering the elderly's context and state. Therefore, the ontology will be described as well as the implementation of user model classes in the IMS architecture.

\section{Materials AND METHODS}

\section{A. GUMO ontology}

GUMO has been the ontology selected to develop the user model as it is the most comprehensive user modeling ontology represented by modern semantic web languages which eases the user model exchange between different applications.

GUMO divides the user model dimensions into three parts: auxiliary, predicate and range. Therefore if one wants to say something about "the user's interest in football", one could divide this into the auxiliary (has interest), the predicate (football) and the range (low-medium-high).

GUMO exposes three main user's dimensions (Basic, Context and Domain) that are modeled within user-adaptive systems and [11]. The Basic User Dimension entails the information related to the physical and psychological user conditions. The classes which are contained in this dimension are: Contact Information, Demographics, Abilities, Personality, Characteristics, Emotional State, Physiological State, Mental State, Motion, Role, Mood, Nutrition, Facial Expression, Relationships and Basic Human Needs. The
Context Dimension defines classes regarding the user's environment or product used as Location, Physical Environment, Social Environment, Sensor Dimensions, Product Information and Travel Contexts. Finally, the Domain Dependent Dimension reflects classes as Interest, Knowledge, and Preference

The auxiliaries employed by GUMO are: hasBelief, hasDone, hasInterest, hasKnowledge, hasLocation, hasPlan, hasPreference, hasRated, hasExperience, hasRegularity, and hasGoal.

\section{B. NGN and IP Multimedia Subsystem Presence service enabler.}

The use of IMS in this work is justified due to its presence service enabler that allows managing user information in order to personalize others services provided. The core of IMS architecture is composed of several servers which handle registered users' information and signaling functions to allow multimedia session management through the Session Initiation Protocol (SIP) [12]. The Presence Service is deployed in IMS architecture through an added value application server, named Presence Server. The SIP/SIMPLE protocol is employed for publishing, subscription and notifying information through events definition by using PUBLISH, SUBSCRIBE and NOTIFY SIP methods. This information is described by means of XML document with PIDF (Presence Information Data Format) format [13] which has been extended with others formats (e.g. RPID, GEOPRIV) with the aim at adding more user information. The presence server can request or update user's profile data in a XML Document Management Server (XDMS) by using the XML Configuration Access Protocol (XCAP). This XDMS server stores user's information which is adjusted to a defined format, known as appusage, which refers to the structure to be followed by user's profile data. The server allows storing as much appusages as type of information needed (contact list, user's images, etc.). Thus, the presence server could offer to $3^{\text {rd }}$ party applications user's current state and user's profile with for inferring the user context.

\section{RESULtS}

\section{A. AALUMO Ontology specification}

Considering the scenarios of AAL services (homecare, health and wellness, supply with goods and chores; safety, security and privacy; social interaction; information and learning; working life; mobility and hobbies), the proposed ontology includes specific classes that characterizes users of 
AAL services. The AALUMO ontology includes some classes and subclasses that belong to GUMO ontology's dimensions, but other classes have been defined to cover as wide as possible the older people conditions and environments.

The Basic Human Needs class is inherited directly from GUMO and expresses the data related to physiological and psychological needs of elderly people. The Preferences class refers to user's preferences in several areas, where their daily activities are developed, and is composed of seven subclasses: interest, nutrition (both from GUMO), privacy, devices, leisure activities, places entertainment and social communities. The Profile class stores user's relevant data which are regular as abilities, contact information, demographics, knowledge, contact persons (all of them extracted from GUMO basic dimension) and chronic diseases. Since elderly people usually suffers any chronic disease, is important to note that we created the subclass chronic disease, not covered by GUMO, that is composed of others subclasses as: disease, limitations (both physical and psychological), medications and threshold medical data parameters. The created Service class is aimed at informing about some characteristics (devices employed, access information like password, and a description) of different AAL services which are usually used by the older person. Considering that our proposed user model is intended to be used in context-aware AAL services, the knowledge about the user's state is essential to infer the context and consequently provide him with the suitable service. Thus, the Current State and Previous State classes refer to aspects which are likely to change in a certain period of time, as emotional state, location, mental state, mood, motion, physical environment, which are extracted from Basic and Context GUMO dimension; and reminders, current device, current medications and current service (developed in this work). Other subclasses as date and time are set in previous state class to note the instant in which the user had that specific state. In addition, the proposed ontology inherits the auxiliaries from GUMO.

\section{B. Overall architecture}

The AAL user model ontology has been defined to be employed by means of the presence service of an IMS architecture. As Fig. 1 shows, the AAL User Model Ontology is deployed in the XDMS server by means of creating appusages according to classes and subclasses defined in the ontology. Any user's device capable of gathering context information is allowed to publish this information using the IMS presence service. Thus, the user's context information will be stored in the presence server. On the one hand, 3rd party applications can subscribe to user's presence infor- mation through a SIP SUBSCRIBE message sent to the presence server. Once the user's presence information has arrived, the presence server will notify about the user's state to the application sending a XML document in a SIP NOTIFY message. Moreover, the presence server will update with the user's presence information the ontology classes related to the user's state (i.e. CurrentState class) using the XCAP PUT method. On the other hand, the applications can access to the user model's information using the XCAP GET method. As the ontology has been deployed through XDMS server's appusages, the user model's information will have a known structure which will be useful to 3rd party application's developer. Thus, the application will be able to apply specific reasoning techniques with the aim at inferring a user's contextual situation and adapting its functions to a certain user when the application has both AAL user model information and presence information.

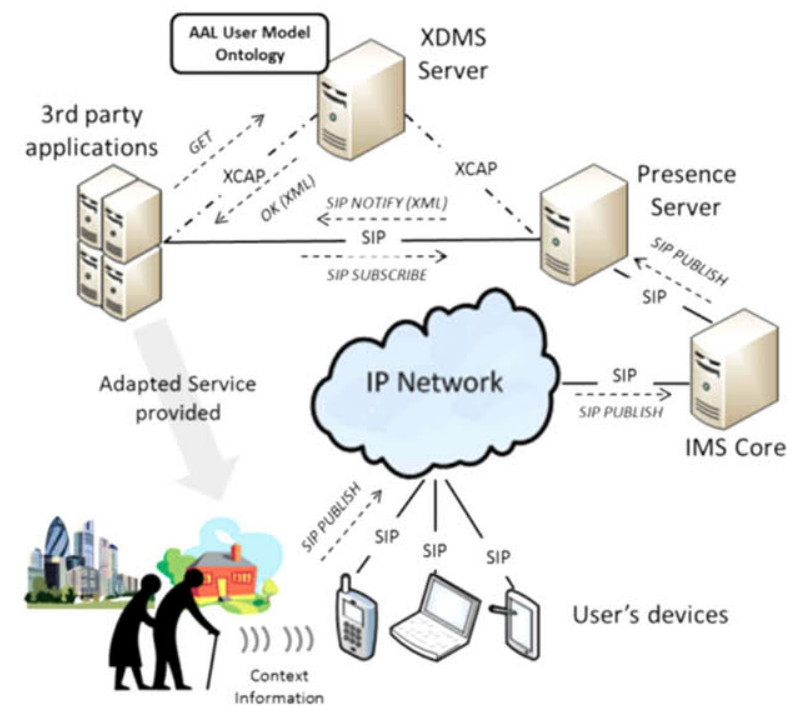

Fig. 1 Overall architecture

\section{AAL User Model appusages}

Regarding the AAL User Model Ontology, the XDMS server will store the user model information which will be based on two appusages: AALUserState and AALUserProfile. The AALUserState appusage refers to the current user's information which is likely to change in a short period of time. The class CurrentState and Service of the AAL User Model Ontology will provide the labels to the AALUserState appusage. An example of a XML document compliant with this appusage format is shown below:

$<$ ns: Userstate

xmlns:ns= ' http: //AALUMO org/schema/AALUserstate

<ns:Emotionalstate> <ns: happiness > </ns:... > 


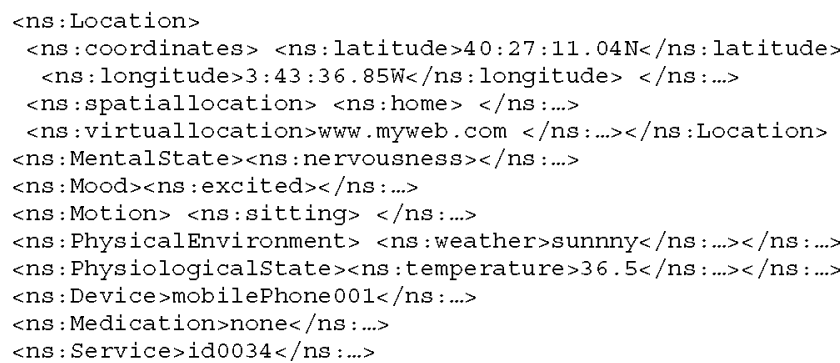

The ontology classes BasicHumanNeeds, and Preferences and Profile are used to create the AALUserProfile appusage that includes the user information which is not going to change in a short period of time. An example of a XML document which shows this appusage is shown as following:

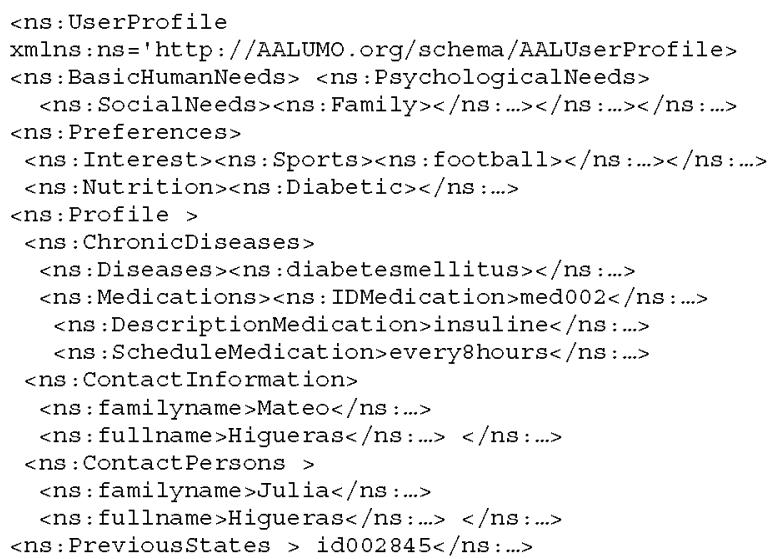

\section{Discussion \& CONCLUSIONS}

Context-awareness services are becoming essential to support the autonomy and independence of elderly people due to the heterogeneity of situations or environment they usually experience. User model and ontologies are very useful to represent user's characteristics and their relationships with environment and applications. On the other hand, the Next-Generation Networks provides enriched multimedia services that allow the context inference considering information from both Internet and mobile spheres. Thus, this work has introduced a user modeling ontology to adapt and personalize Ambient Assisted Living services provided through NGN networks. By using the Presence service, 3rd party applications can access to user's presence and user profile information with the aim at applying context reasoning techniques. Therefore, two appusages has been defined formatting the information contained in the AAL user model ontology presented.

As future work, the ontology could be extended including as much requirements (i.e. extended contact infor- mation, or more user's devices and services characteristics) as AAL services may meet in order to support the elderly people's daily life. Furthermore, the AALUMO ontology could also support the definition of a context service enabler with context management capabilities that could relieve 3 rd party applications of context managing tasks.

\section{AcKNOWLEDGMENTS}

We would like to acknowledge for contributing to this work to Liss Hernandez.

\section{REFERENCES}

[1] Ambient Assisted Living at http://www.aal-europe.eu/

[2] Y. Cao, L. Tao, and G. Xu, "An event-driven context model in elderly health monitoring," in Ubiquitous, Autonomic and Trusted Computing, 2009. UIC-ATC'09. Symposia and Workshops on, 2009, pp. $120-124$.

[3] F. Paganelli and D. Giuli, "An Ontology-Based System for ContextAware and Configurable Services to Support Home-Based Continuous Care," IEEE Transactions on Information Technology in Biomedicine, vol. 15, no. 2, pp. 324-333, Mar. 2011.

[4] D. Heckmann, T. Schwartz, B. Brandherm, M. Schmitz, and M. von Wilamowitz-Moellendorff, "Gumo-the general user model ontology," in User modeling 2005, Springer, 2005, pp. 428-432.

[5] P. Gutheim, "An ontology-based context inference service for mobile applications in next-generation networks," Communications Magazine, IEEE, vol. 49, no. 1, pp. 60-66, 2011.

[6] Y. Evchina, A. Dvoryanchikova, and J. L. M. Lastra, "Ontological framework of context-aware and reasoning middleware for smart homes with health and social services," in Systems, Man, and Cybernetics (SMC), 2012 IEEE International Conference on, 2012, pp. 985-990.

[7] D. Preuveneers, J. Van den Bergh, D. Wagelaar, A. Georges, P. Rigole, T. Clerckx, Y. Berbers, K. Coninx, V. Jonckers, and K. De Bosschere, "Towards an extensible context ontology for ambient intelligence," in Ambient intelligence, Springer, 2004, pp. 148-159.

[8] C. Baladron, J. M. Aguiar, B. Carro, L. Calavia, A. Cadenas, and A. Sanchez-Esguevillas, "Framework for intelligent service adaptation to user's context in next generation networks," Communications Magazine, IEEE, vol. 50, no. 3, pp. 18-25, 2012.

[9] J. Kim, J. Jeong, S. Nam, and O. Song, "Intelligent Service Enabler Based on Context-Aware in Next Generation Networks," 2008, pp. 802-806.

[10] A. Moon, Y. Park, and S. Kim, "Higher order knowledge management platform for the personalized services in next generation networks," in Networked Digital Technologies, 2009. NDT'09. First International Conference on, 2009, pp. 452-457.

[11] UbisWorld web page: http://ubisworld.ai.cs.uni-sb.de/index.php

[12] Y. ITU-T, General overview of NGN. December, 2004.

[13] H. Sugano et al. "Presence Information Data Format (PDIF)" IETF RFC 3863, 2004.

Author: Pedro Antonio Moreno Sánchez

Institute: Bioengineering and Telemedicine Group- Technical University of Madrid

Street: E.T.S.I. Telecomunicación. Avda Complutense 30,

City: Madrid

Country: Spain

Email: pmoreno@gbt.tfo.upm.es 